\title{
Increased IL-6 secretion by aged human mesenchymal stromal cells disrupts hematopoietic stem and progenitor cells' homeostasis
}

\author{
Kelsey O'Hagan-Wong ${ }^{1}$, Stéphanie Nadeau ${ }^{2}$, Audrey Carrier-Leclerc ${ }^{2}$, Felipe \\ Apablaza ${ }^{1}$, Reggie Hamdy ${ }^{3}$, Dominique Shum-Tim ${ }^{4}$, Francis Rodier ${ }^{2,5}$ and Inés \\ Colmegna ${ }^{1}$ \\ ${ }^{1}$ Division of Rheumatology, Department of Medicine, McGill University, Montreal, QC, Canada \\ ${ }^{2}$ CRCHUM and Institut du Cancer de Montréal, Montreal, QC, Canada \\ ${ }^{3}$ Shriners Hospital for Children and Division of Orthopedic Surgery, McGill University, Montreal, QC, Canada \\ ${ }^{4}$ Divisions of Cardiac Surgery and Surgical Research, McGill University, Montreal, QC, Canada \\ ${ }^{5}$ Department of Radiology, Radio-Oncology and Nuclear Medicine, Université de Montréal, Montreal, QC, Canada \\ Correspondence to: Inés Colmegna, email: ines.colmegna@mcgill.ca \\ Francis Rodier, email: rodierf@mac.com
}

Keywords: mesenchymal stromal cells, hematopoietic stem and progenitor cells, aging, senescence, senescence-associated secretory phenotype, Gerotarget

Received: January 06, $2016 \quad$ Accepted: January 30, 2016

Published: February 24, 2016

\section{ABSTRACT}

Hematopoietic stem and progenitor cell (HSPC) homeostasis declines with age, leading to impaired hematopoiesis. Mesenchymal stromal cells (MSC) are critical components of the bone marrow niche and key regulators of the balance between HSPC proliferation and quiescence. Accrual of DNA damage, a hallmark of cellular aging, occurs in aged MSC. Whether MSC aging alters the bone marrow niche triggering HSPC dysfunction is unknown. Using a human MSC-HSPC co-culture system, we demonstrated that DNA damaged MSC have impaired capacity to maintain CD34 ${ }^{+}$CD $38^{-}$ HSPC quiescence. Furthermore, human MSC from adult donors display some hallmarks of cellular senescence and have a decreased capacity to maintain HSPC quiescence and the most primitive CD34 ${ }^{+}$CD 38 - subset compared to MSC from pediatric donors. IL-6 neutralization restores the MSC-HPSC crosstalk in senescent and adult MSC-HSPC co-cultures highlighting the relevance of the local microenvironment in maintaining HSPC homeostasis. These results provide new evidence implicating components of the MSC secretome in HSPC aging.

\section{INTRODUCTION}

Hematopoietic stem and progenitor cells (HSPC) sustain the normal turnover of blood cells throughout an individual's lifetime. The regulation of HSPC function is a complex process that involves the integration of HSPC inherent programs with signals originating from components of the bone marrow (BM) niche [1]. These signals ensure the balance between HSPC quiescence, to protect the stem cell pool from premature exhaustion, and HSPC cycling which is required to accomplish efficient hematopoiesis [2].

Central to the regulation of HSPC quiescence are the multifaceted interactions of HSPC with key cellular components of the BM niche, among them multipotent mesenchymal stromal cells (MSC) [3]. MSC contribute to the HSPC microenvironment through complex cellcell interactions and the secretion of hematopoietins that regulate $\mathrm{HSPC}$ proliferation, differentiation, and migration $[3,4,5,6]$.

Recent evidence in myelodysplastic syndromes suggests that BM-MSC senescence could impact HSPC homeostasis [7] and that targeting senescent cells in animal models rejuvenate aged HSPC [8]. Cellular senescence is a vital tumor suppression mechanism also involved in aging and tissue repair [9]. The accumulation of senescent cells in premature aging models directly contributes to age-associated organ dysfunctions [6], probably owing to 
their senescence-associated secretory phenotype (SASP), a potent pro-inflammatory microenvironment remodeler [10]. Whether MSC senescence during aging impacts the bone marrow microenvironment remains undefined. Given that DNA damage is a hallmark of cellular senescence and aging $[9,11]$, we tested the impact of MSC irradiationinduced cellular senescence on the modulation of HSPC homeostasis. We demonstrate that DNA damage induces a classical senescent phenotype in MSC including the production of high levels of IL-6 that in turn mediates the reduced capacity to preserve HSPC quiescence and to maintain immature $\mathrm{CD} 34^{+}$subpopulations. Furthermore, our data suggest that MSC from older donors, despite of not harboring a complete senescent phenotype behave functionally as senescent cells secreting increased levels of IL-6 that alters HSPC biology.

\section{RESULTS}

All adipose derived MSC used in this study met the minimal criteria proposed by the ISCT [12] including a spindle shaped morphology and the expression of positive MSC markers in more than 95\% of cells (CD73 CD105, and CD90) without lineage commitment markers (CD34 CD19 CD14 and HLADR) (Supplementary Figure 1A). At the end of passage 3 , all cells differentiated in vitro to osteoblasts, adipocytes and chondrocytes (Supplementary Figure 1B).

\section{DNA damage induces MSC senescence}

We have previously shown that DNA double strand breaks induce hallmarks of cellular senescence in human fibroblasts and endothelial cells including the activation of a persistent DNA damage response (DDR) [13, 14]; and that the accumulation of DNA damage is a salient feature of cellular aging $[9,11]$. To determine whether DNA damage is sufficient to drive a complete cellular senescence phenotype in MSC, we first treated MSC with escalating doses of gamma irradiation and determined whether these cells underwent permanent growth arrest, a hallmark of cellular senescence [11]. MSC proliferation was evaluated 8 days post irradiation using quantitative LI-COR analysis of total cellular DNA (Figure 1A). We observed a dose-dependent decrease in proliferation that leveled around 5Gy, suggesting that this represents the minimal dose that generated prolonged senescence-like MSC growth arrest. To characterize other senescenceassociated hallmarks in MSC irradiated with $5 \mathrm{~Gy}$, we first measured senescence-associated beta galactosidase (SABG) activity, the most widely used senescence biomarker. SABG activity was detected in damaged MSC or fibroblasts 9 days following irradiation while no detectable SABG expression was found baseline (0 Gy) (Figure 1B). We also observed that irradiated MSC lost their spindle shape morphology and acquired a typical senescence-associated enlarged and flattened phenotype. This was confirmed by a significant increase in cell size 8 days after irradiation as measured by flow cytometry (Figure 1C).

\section{Irradiated MSC express molecular markers of cellular senescence}

Cellular senescence is characterized by a series of hallmarks including the activation of a persistent DDR [11, 14, 15]. Following DNA double-strand break (DSB), a series of chromatin modifications triggered by the DDR kinase ATM can be visualized in the damaged nuclei as DNA damage foci. Markers of DNA damage foci include the local phosphorylation of histone variant $\mathrm{H} 2 \mathrm{AX}$ at serine 139 residue $(\gamma \mathrm{H} 2 \mathrm{AX})$ and the recruitment of the damaged chromatin reader 53BP1 (p53 binding protein 1) $[13,16]$. We performed immunofluorescence on non-irradiated controls and irradiated MSC and readily observed the appearance of colocalized nuclear $\gamma \mathrm{H} 2 \mathrm{AX} / 53 \mathrm{BP} 1$ foci in irradiated cells (Figure 1D-1E). The tumor suppressor PML (promyelocytic leukemia) is also part of the DDR and an important modulator of p53 that is consistently up-regulated in senescent cells [17]. Immunoflurescence targeting PML revealed a strong increase of the protein in MSC, 10 days after 5Gy irradiation (Figure 1F). When DNA damage foci remains unrepaired for more than 48 hours following damage induction, they are converted to a structure termed DNASCARS, which is revealed by their juxtaposition to another nuclear domain, the PML nuclear bodies or PMLNBs [14]. We evaluated the presence of DNA-SCARS in irradiated MSC by detecting co-localization between $\gamma \mathrm{H} 2 \mathrm{AX}$ or 53BP1 and PML-NBs and found a consistent increase in damaged cells (Figure $1 \mathrm{G}-1 \mathrm{H}$ and summary data in Figure 1I). Overall, irradiated MSC display all the critical hallmarks associated with the presence of a senescence-associated persistent DDR.

Finally, to evaluate the ability of DNA damageinduced senescent MSC to impact the microenvironment, we measured the secretion of two highly conserved components of the SASP: the pro-inflammatory cytokines IL-6 and IL-8 (Figure 2) [10]. The secretion of IL-6 and IL- 8 by MSC consistently nearly doubled ( $p=0.004$ ) after irradiation although there was a large variability in the baseline cytokine production associated to individual donors. Overall the fold increase for IL-6 secretion in all paired samples (pre-IR vs. post-IR) ranged from 1.11 to 2.92 for an average increase of 1.95 fold while IL-8 secretion increased within a range of 1.07 to 5.40 for an average of 2.61 fold.

Thus, irradiation-induced DNA damage promotes a classical cellular senescence phenotype in MSC including the expression of a SASP $[10,13,18]$. 
A

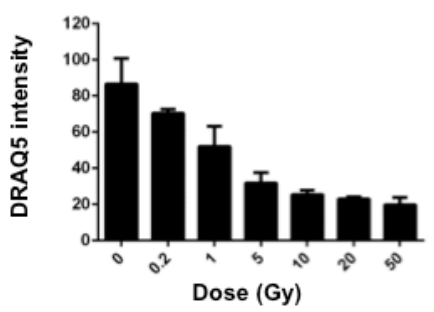

C

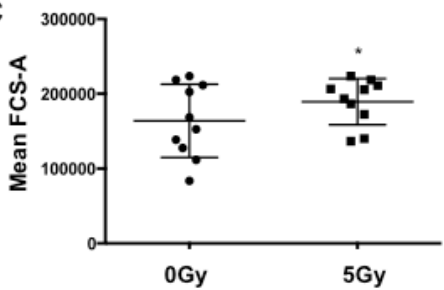

D
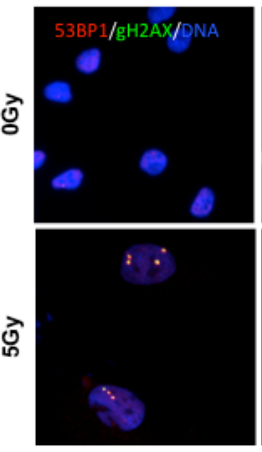

G
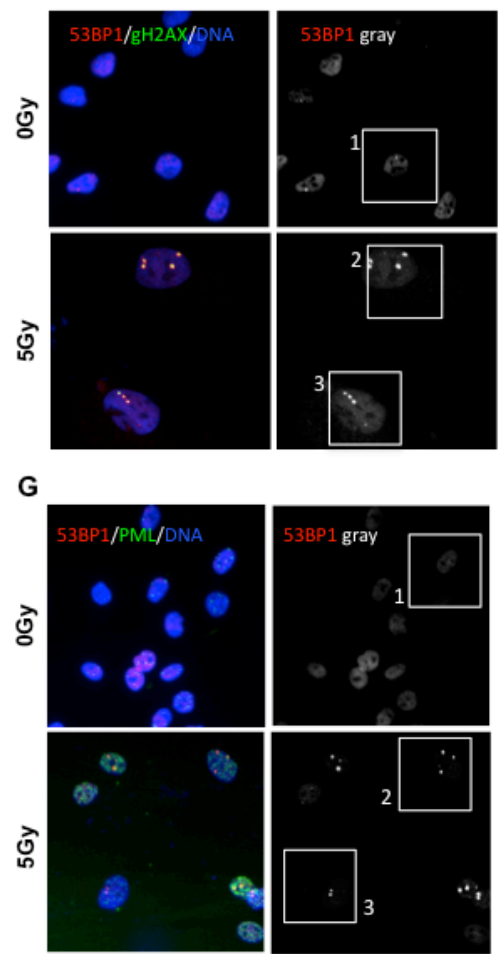

B
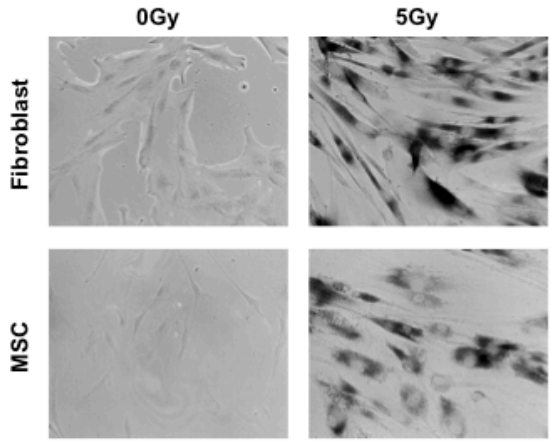

E

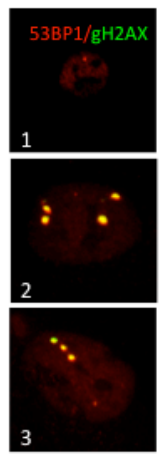

H

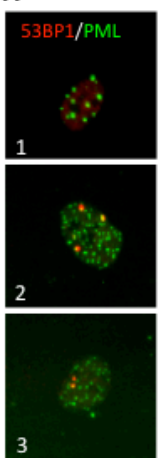

F
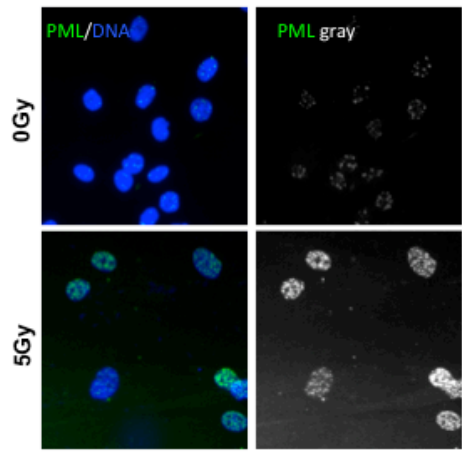

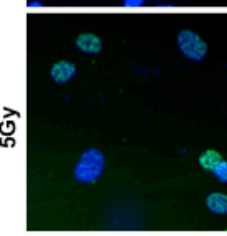

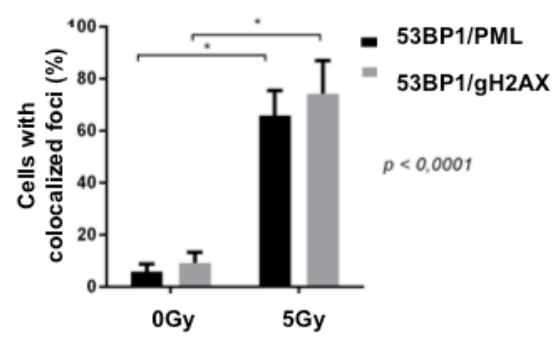

Figure 1: Irradiation induces senescence biomarkers in MSC. A. MSCs were exposed to increasing doses of radiation $(0$ - 50 Gy) and proliferation was assessed 8 days later using LI-COR analysis (DRAQ5 fluorescence intensity (total DNA content). B. Senescenceassociated beta-galactosidase activity was assessed at baseline and 9 days following 5 Gy irradiation in fibroblasts (positive controls, HCA2-hTERTs) and MSCs. C. MSC cell size was evaluated 8 days following 5 Gy irradiation using FACS. D. Direct molecular markers of DNA double strand breaks were evaluated in control and irradiated MSC using immunofluorescence (9 days following irradiation with 5 Gy). Representative images of cells harboring small nuclear DNA damage foci constituted of both 53BP1 (red) and phospho-H2AX (green) (yellow highlight red-green colocalization, the nuclei are counterstained in blue (DAPI)). The extracted 53BP1 red channel is presented in grayscale to highlight 53BP1 DNA damage foci that appears like nuclear white dots (right panels). Selected cells are boxed in white squares labeled 1-3 for magnification in E. to highlight colocalization between 53BP1 (red) and phospho-H2AX (green). Note the yellow dots in irradiated cells (box 2 and 3). F. The molecular biomarker of senescence (PML, green) was evaluated in control and irradiated MSC using immunofluorescence (nuclei counterstained in blue (DAPI)). Notice increased total levels of PML and PML nuclear bodies in irradiated cells. The extracted PML green channel is presented in grayscale to highlight the nuclear increase in PML levels (right panels) G. Representative images of cells harboring DNA-SCARS highlighted by 53BP1 (red) and PML (green) colocalization. Nuclei are counterstained in blue (DAPI). The extracted 53BP1 red channel is presented in grayscale to highlight 53BP1 DNA damage foci that appears like nuclear white dots (right panels). Selected cells are boxed in white squares labeled 1-3 for magnification in $\mathbf{H}$. to highlight colocalization (yellow) between 53BP1 (red) and PML (green). Note the yellow dots in irradiated cells (box 2 and 3). I. The quantified data for the representative images presented for DNA damage foci (53BP1-gH2AX colocalization) and DNA-SCARS (53BP1PML colocalization) is presented as the percentage of cells with $>1$ colocalized nuclear foci. Mean \pm SD of 4 independent experiments are reported where $(*)$ represents $p \leq 0.0001$. 


\section{MSC senescence leads to decreased HSPC quiescence in vitro}

Senescent cells have the capacity to modulate their microenvironment $[10,11]$. Given the critical role that MSC play in preserving normal HSPC function over time, we sought to investigate how MSC senescence might impact their capacity to support HSPC in vitro. To test this we co-cultured HSPC for 4 days with either non-irradiated MSC (controls) or irradiated MSC (senescent or iMSC) (Figure 3). To control for MSC death and to ensure that equal numbers of MSC were plated in irradiated and nonirradiated co-culture conditions, MSC were counted and re-plated 48 hours following irradiation.

Senescent MSC induced significantly higher $\mathrm{CD}^{2}{ }^{+}$expansion compared to controls (expansion index $3.12 \pm 1.31$ vs. $2.56 \pm 1.21, p=0.002$ ) (Figure $3 \mathrm{~A})$. The analysis of $\mathrm{CD} 4^{+}$proliferation also revealed that senescent MSC maintained a significantly lower proportion of $\mathrm{CD}_{3} 4^{+}$in generation 0 compared to nonirradiated MSC (33.11 \pm 22.35 vs $41.25 \pm 26.70, p=0.01)$ (Figure 3B). Moreover, senescent MSC maintained a significantly lower proportion of $\mathrm{CD} 34^{+} \mathrm{CD} 38^{-}$cells compared to non-irradiated MSC $(3.99 \pm 1.8 v s .7 .37 \pm \mathrm{v}$ $5.99, p=0.02$ ) (Figure 3C). Of relevance, CD34+ viability was not different $(p=0.4)$ in irradiated and non-irradiated MSC-HSPC co-cultures (Figure 3D). To test whether soluble factors mediated the increased HSPC expansion in iMSC-HSPC co-cultures, we performed supernatant transfer experiments. Conditioned medium from senescent MSC induced significantly higher HSPC expansion compared to conditioned medium from control MSC (1.1 \pm 0.1 vs. $1.3 \pm 0.2$ ) (Figure 3E).

Largely, these results imply that MSC senescence induced by DNA damage alters the MSC capacity to support HSPC in vitro at least in part via secreted factors. Specifically, senescent MSC are less capable to maintain $\mathrm{CD}_{3} 4^{+}$quiescence and to preserve the $\mathrm{CD} 34^{+} \mathrm{CD} 38^{-}$ subpopulation.

\section{MSC from adult donors display senescence markers}

The wide range of inflammatory cytokine secretion by non-irradiated MSC samples (Figure 2) suggested a potential association with the donors' age. To determine whether MSC undergo cellular senescence as part of the normal aging process in vivo, we comparatively examined the expression of senescence biomarkers in passage 3 (P3) MSC isolated from pediatric (age $<16$ years) and adult (age $>40$ years) patients. Adult MSC not only had a larger size but also displayed reduced proliferation rates compared to their pediatric counterparts (Figure 4A-4B). Probing the SASP also revealed that adult MSC produced significantly higher levels of the senescence-associated cytokines IL-6 and IL-8 compared to pediatric MSC (Figure 4C-4D). In fact, the difference in IL-6 or IL-8 $(p=0.0001)$ secretion between adult and pediatric MSC was much higher than what was observed in any samples following irradiation (IL-6 increase in adults: 5.46 fold; IL-8 increase 5.89 fold). However, consistent with what was previously reported in human bone marrow [19] and adipose MSC [20] in elderly individuals, we found low to undetectable levels of SABG (below 10\%) in all early passage cultures tested, irrespective of their age (data not shown). This suggests that adult MSC display some, but not all senescence hallmarks.

\section{Adult MSC have a decreased capacity to maintain primitive $\mathrm{CD34}^{+} \mathrm{CD}^{-} 8^{-}$subpopulations and HSPC quiescence}

We next determined the impact of the chronological aging-induced senescent-like phenotype of MSC on their capacity to support HSPC. We co-cultured CD34 cells from a single donor with MSC from adult or pediatric donors (Figure 5). We found that adult MSC induced higher overall $\mathrm{CD} 34^{+}$expansion compared to pediatric MSC co-cultures (expansion index $2.49 \pm 0.960$ vs. $1.83 \pm 0.57 ; p=0.04$ ) (Figure $5 \mathrm{~A}$ ). The frequency of $\mathrm{CD}^{+} 4^{+}$cells in generation 0 was decreased in the adult compared to pediatric MSC co-cultures $(49.57 \pm 13.89 \%$ vs. $61.20 \% \pm 15.46 ; p=0.04$ ) (Figure 5B). Furthermore, adult MSC have a reduced capacity to maintain $\mathrm{CD} 34^{+} \mathrm{CD} 38$ subpopulations compared to pediatric MSC (4.6 $\pm 3.3 \mathrm{vs.}$ 9.7 $\pm 6.8 ; p=0.02$ ) (Figure 5C). No differences were observed in $\mathrm{CD}^{+} 4^{+}$cell viability between adult and pediatric MSC cultures $(p>0.5)$ (Figure 5D).

Taken together, these results reveal important functional differences between adult and pediatric MSC. In particular, consistent with their senescentlike characteristics, adult MSC display a decreased capacity to maintain $\mathrm{CD} 34^{+}$quiescence and $\mathrm{CD} 34^{+} \mathrm{CD} 38^{-}$ subpopulations compared to pediatric MSC.

\section{IL-6 reduces HSPC quiescence in adult MSC co- cultures}

Given the elevated levels of secreted IL- 6 by both irradiated and adult MSC and the relevance of IL- 6 as a hematopoietin [21], we hypothesized that IL-6 played a role in promoting $\mathrm{CD} 34^{+}$expansion. To test this, we treated with an IL-6 neutralizing antibody iMSC and control co-cultures (Figure 6A-6C), and adult and pediatric MSC co-cultures (Figure 6D-6F).

The addition of anti-IL- 6 to senescent MSC-HSPC co-cultures reduced the $\mathrm{CD} 34^{+}$expansion from $1.2 \pm 0.19$ to $0.97 \pm 0.22$ ( $p=0.004$ Figure $6 \mathrm{~A}$, values normalized to those of HSPC in non-irradiated co-cultures). IL-6 neutralization also increased the percentage of $\mathrm{CD} 34^{+}$cells 
A

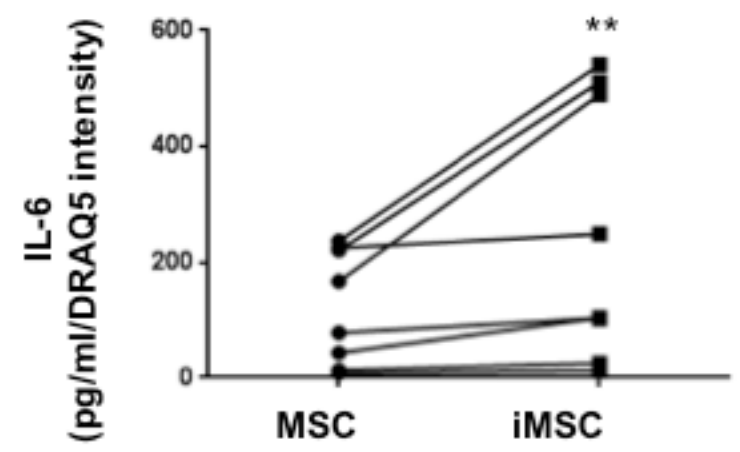

B

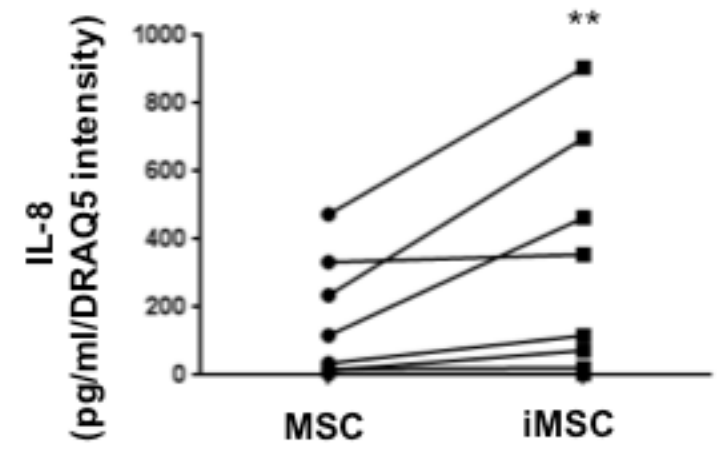

Figure 2: Increased secretion of senescence-associated cytokines by irradiated MSC. The production of IL-6 and IL- 8 in iMSC (5Gy) and controls was evaluated by ELISA 9 days after irradiation. iMSC produced significantly higher levels of IL-6 A. and IL-8 B. compared to controls. Mean (triplicate) of 7 independent experiments are reported where (**) represents $p \leq 0.01$.
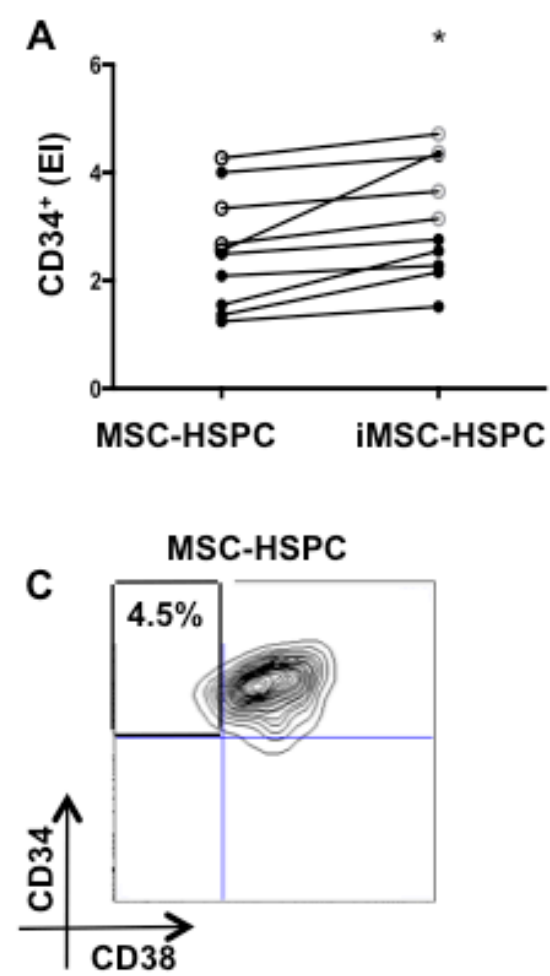

D

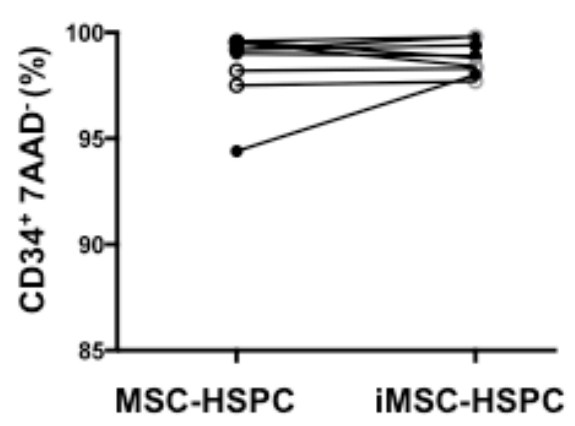

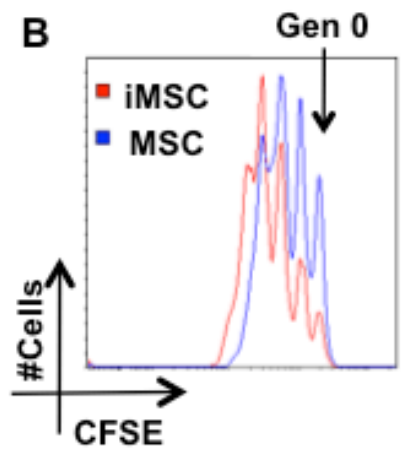

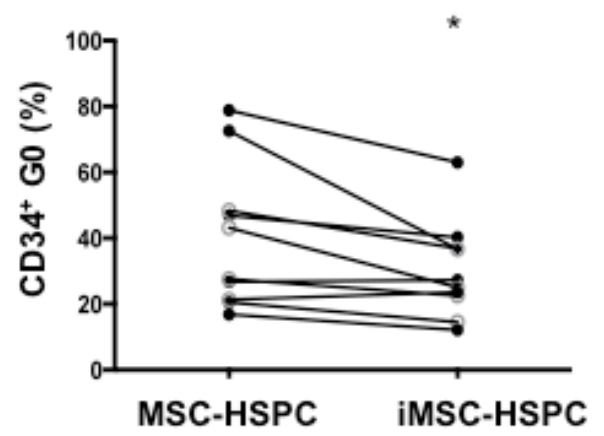

\section{iMSC-HSPC}
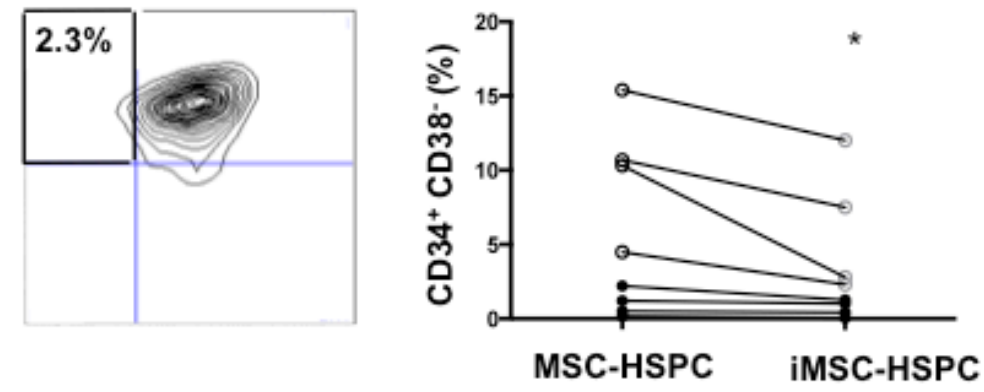

E

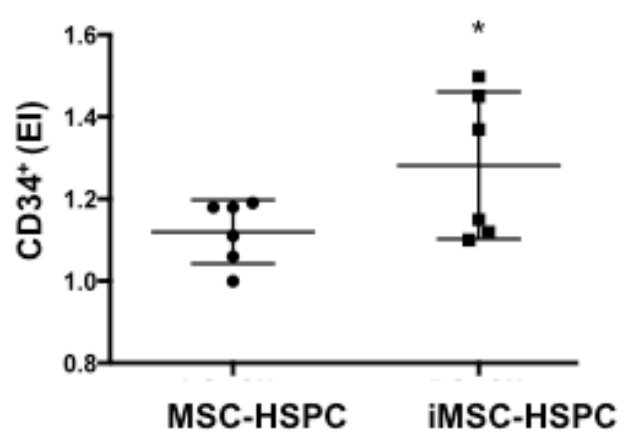

Figure 3: Senescent MSC reduce HSPC quiescence and the frequency of primitive $\mathrm{CD}^{-} 4^{+} \mathrm{CD}^{-} 8^{-}$cells. $\mathrm{A}$. $\mathrm{CD}^{+} 4^{+}$expansion, B. percentage of $\mathrm{CD}_{4} 4^{+}$in generation 0 , C. frequencies of CD34 ${ }^{+} \mathrm{CD} 38^{-}$subpopulations, and $\mathbf{D}$. CD $34^{+}$viability were evaluated in MSCHSPC and iMSC-HSPC (5Gy) co-cultures (1:1 ratio) following 4 days of co-culture. Open-faced circles represent MSC from pediatric donors $\left(<16\right.$ years-old) and black circles represent MSC from adult donors ( $>40$ years-old). E. CD34 ${ }^{+}$proliferation was also evaluated following HSPC culture with conditioned medium from MSC and iMSC. Mean \pm SD of 6-10 independent experiments are reported where (*) represent $p \leq 0.05$. 
in generation 0 (Figure 6B) and increased the frequency of $\mathrm{CD} 34^{+} \mathrm{CD} 38^{-}$cells to levels that were similar to control conditions (Figure 6C). The addition of anti-IL6 to cultures however did not impact CD34 $4^{+}$viability (data not shown).

Similarly, blocking IL-6 in adult MSC co-cultures caused a significant decrease in $\mathrm{CD}_{3} 4^{+}$expansion $(2.49 \pm 0.96 v s$ 1.93 \pm 0.67$)$ (Figure 6D) and restored the proportion of CD34 $4^{+}$cells in generation $0(49.57 \pm 13.89$ vs 58.56 \pm 15.50 ) (Figure 6E). Those levels were not significantly different to those of pediatric co-cultures (expansion index: $1.88 \pm 0.58$, percentage in generation 0 : $61.20 \pm 15.46)$. The addition of IL-6 antibody to pediatric co-cultures however had no significant effect on CD34 proliferation or quiescence. Blocking IL-6 tend to increase the percentage of $\mathrm{CD} 34^{+} \mathrm{CD} 38^{-}$subpopulations in adult co-cultures and tend to decrease that subpopulation in pediatric co-cultures, albeit differences were not

A
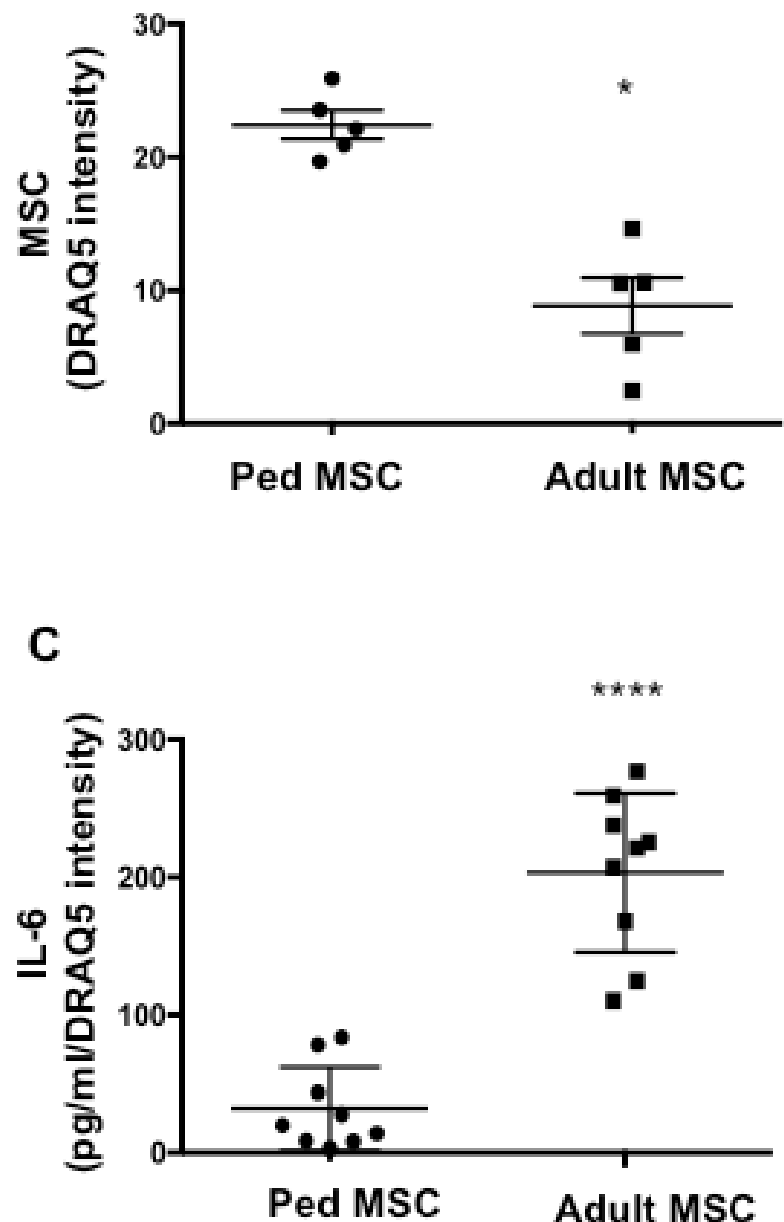

significant $(p=0.25)$ (Figure 6F). The addition of antiIL-6 to adult and pediatric co-cultures had no significant effect on CD $34^{+}$viability.

These results suggest that increased IL-6 production by damaged or old MSC in co-cultures disrupts CD34 quiescence. The capacity to maintain $\mathrm{CD} 34$ quiescence, but not $\mathrm{CD} 34^{+} \mathrm{CD} 38^{-}$populations, in adult co-cultures can be restored to pediatric levels by antagonizing IL-6.

\section{DISCUSSION}

Hematopoietic stem cell function declines with age [22]. Given that signals from the bone marrow niche are key for normal hematopoiesis it has been proposed that age associated changes in the bone marrow niche contribute to HSPC aging [23]. Although there is an age-related decline in MSC immunopotency [24, 25];

B
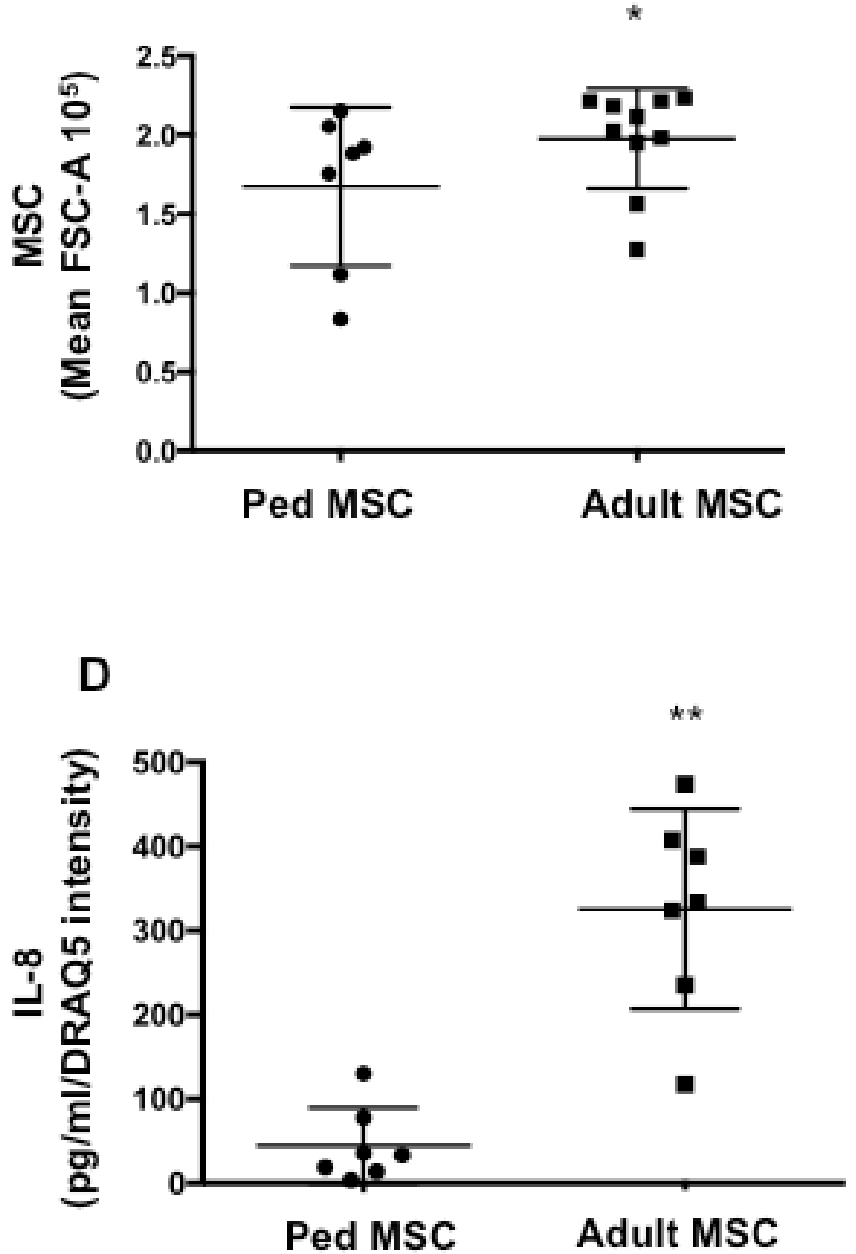

Figure 4: MSC from adult donors have a senescence-like phenotype. A. MSC were isolated from adult ( $>40$ years) and pediatric donors (<16 years). MSC proliferation was measured by DRAQ5 intensity day 4 post seeding in adult and pediatric samples. B. Cell size was measured by flow cytometry (Mean FSC-A) in adult and pediatric MSC samples. C. IL-6 and D. IL-8 production by adult and pediatric MSC were quantified by ELISA. Mean \pm SD of 7-10 independent experiments are reported where $\left(^{*}\right)$ represents $p \leq 0.05$. 
it is unknown to what extent those age-related MSC chronological changes impact the microenvironment and HSPC function. This study provides direct evidence that adult MSC show hallmarks of senescence, in particular increased secretion of SASP factors, which alter HSPC homeostasis in vitro.

Our results indicate that much like other cell types, irradiation induces typical senescence hallmarks in MSC including growth arrest, SABG expression, and increase in cell size. These findings are consistent with previous studies looking at similar readouts in BM-MSC in response to irradiation $[26,27]$. We further characterized MSC senescence by evaluating the presence of persistent DDR signaling and two conserved pro-inflammatory cytokines of the SASP. Consistent with a recent study that analyzed BM-MSC, we found that IL-6 and IL-8 secretion is increased in MSC in response to DNA damage (2-3 fold higher in iMSC compared to non-irradiated) [27]. The SASP influences cell migration, adhesion, and specifically in BM-MSC the upregulation of IL-6 in response to replicative senescence has been linked to a loss of stemness [28].

We next assessed the effects of MSC senescence on the MSC-HSPC crosstalk. Stem cell quiescence, protects HSC from accumulation of molecular damage, including the induction of replication associated DNA damage, oxidative-stress-induced damage, and telomere shortening [29]. MSC from various sources maintain $\mathrm{CD} 34^{+}$in an undifferentiated state ex vivo and this ability seems to be affected by MSC aging, as suggested by the fact that
A

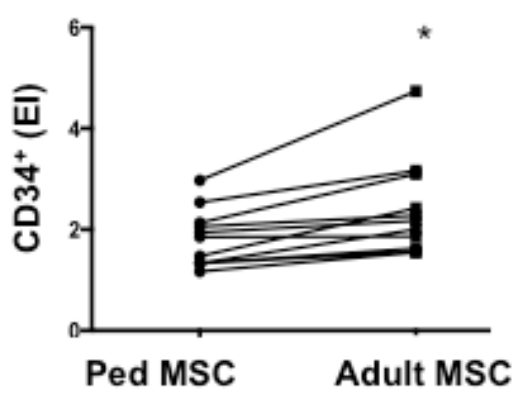

Ped MSC

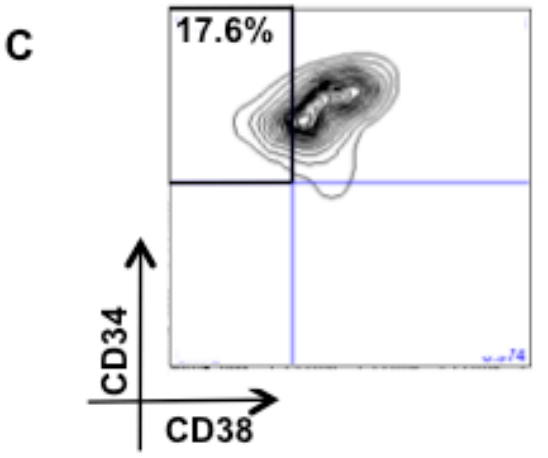

B

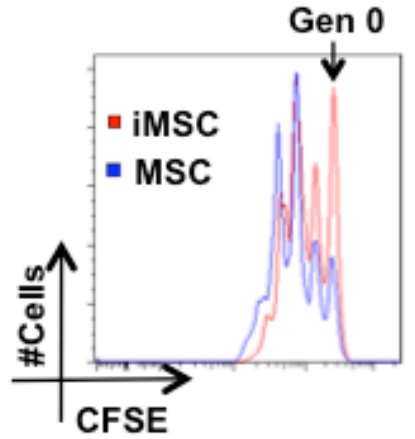

Adult MSC

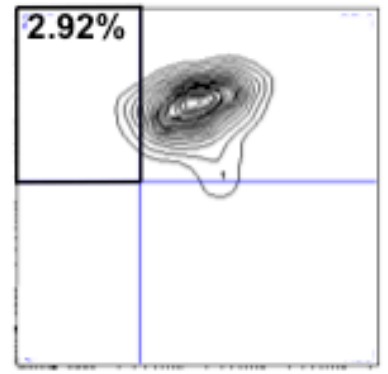

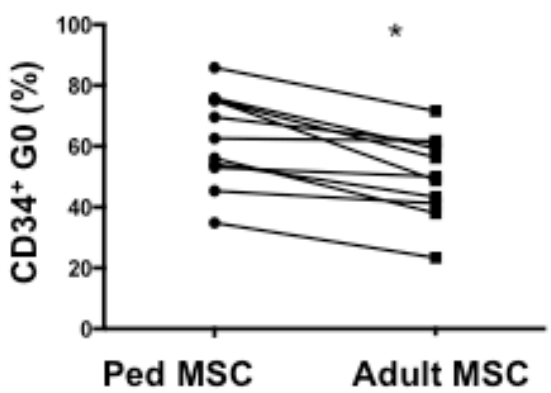

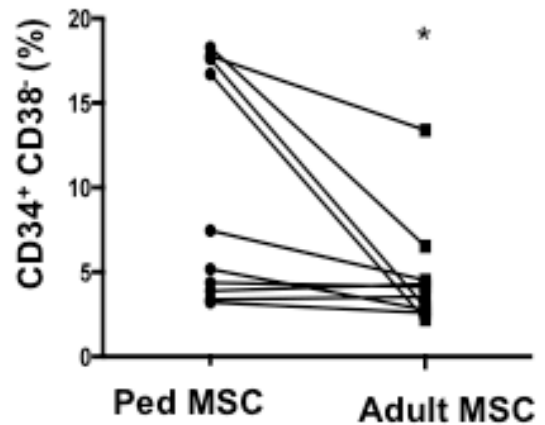

D

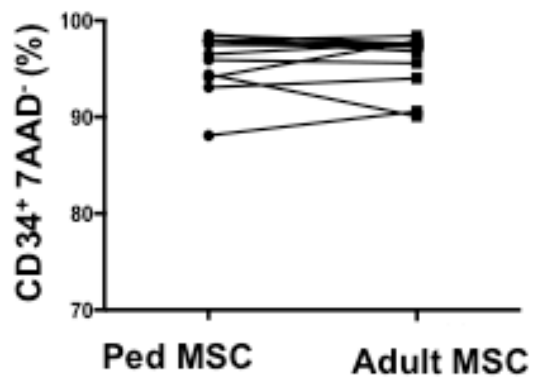

Figure 5: Loss of HSPC quiescence and primitive subpopulations in adult MSC-HSPC co-cultures. A. CD34 ${ }^{+}$expansion, B. percentage of $\mathrm{CD}_{4} 4^{+}$in generation 0 , C. frequency of $\mathrm{CD} 34^{+} / \mathrm{C} 38^{-}$subpopulations, and $\mathbf{D}$. viability were evaluated in HSC-MSC cocultures (1:1 ratio) at day 4 . MSC used were isolated from pediatric ( $<16$ years) or adult ( $>40$ years) donors. Mean of 10 independent experiments are reported where $(*)$ represents $p \leq 0.05$. 
Terc $^{-/-}$MSC cells show decreased ability to maintain HSC [30]. We found that MSC support viable CD34+ cells preserving the $\mathrm{CD} 34^{+} \mathrm{CD} 38^{-}$subset which has the highest reconstitution capacity [31]. Senescence decreased the MSC capacity to promote HSPC quiescence reducing the frequency of $\mathrm{CD} 34^{+} \mathrm{CD} 38$. This may reflect a shift from HSPC self-renewal towards HSPC proliferation and differentiation which ultimately could lead to stem cell pool replicative senescence and increase in genome aberrations [32].

To evaluate whether chronological aging recapitulates hallmarks of DNA damage induced MSC senescence, we compared the phenotype of adult and pediatric MSC and tested their HSPC supportive ability. Adult MSC have a "senescence-like" phenotype displaying only certain classical markers of cellular senescence. Compared to pediatric MSC, the adult MSC size is larger [33] and they secrete 4-times higher levels of IL-6 and IL-8. Furthermore, consistent with previous reports, other markers of senescence, specifically SABG activity was absent in early passage adult and pediatric MSC [19, 20].

While adult MSC did not display all of the classical features of cellular senescence, we demonstrate for the first time that adult MSC functionally behaved similarly to senescent MSC. Like irradiated MSC co-cultures, adult MSC promoted $\mathrm{CD}_{3} 4^{+}$expansion while retaining fewer $\mathrm{CD} 4^{+}$in generation 0 and decreased the frequency of primitive $\mathrm{CD} 34^{+} \mathrm{CD} 38^{-}$cells. These results would favor the use of pediatric MSC over adult MSC for the ex vivo expansion of $\mathrm{CD} 34^{+}$cells. Alternatively, our results also raise the possibility that the manipulation of senescent MSC in vivo via pharmaceutical inhibition of SASP factors like IL-6 or through targeted elimination of these cells, as is now performed in mouse models, could be used in the context of natural aging or of premature aging syndromes [6, 8, 34, 35, 36, 37].

In the context of aging, we hypothesize that an altered HSPC-MSC cross-talk can promote HSPC proliferation resulting in a functional exhaustion of the hematopoietic system. This negative impact of microenvironment senescence or aging on the regulation of HSPC quiescence was previously suggested following an in vitro evaluation of MSC replicative senescence demonstrating that early passage MSC favor HSPC selfrenewal whereas culture-aged late passage MSC favor differentiation and expansion of HSPC [38]. In vivo, the aging of the stem cell niche in a different context has also been shown to disrupt satellite muscle cells quiescence leading to a decline in regenerative capacity
A

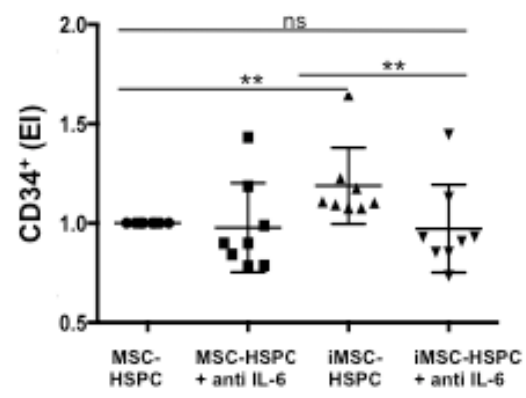

D

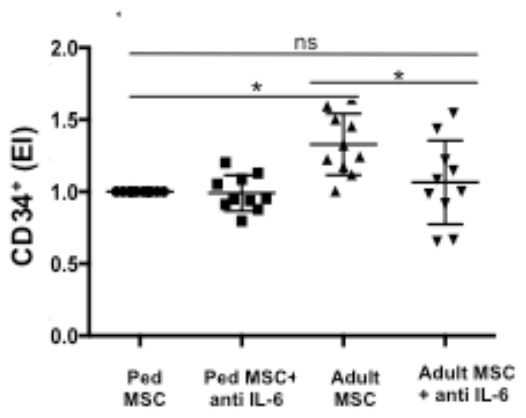

B

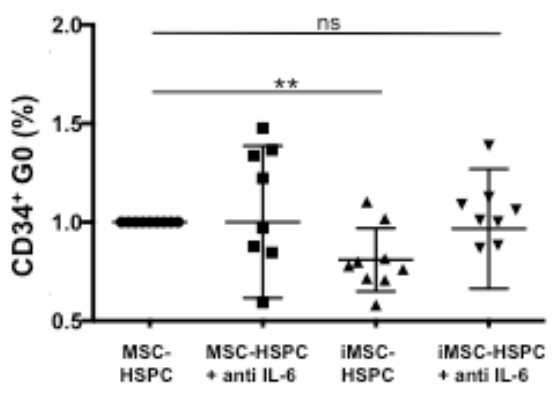

E

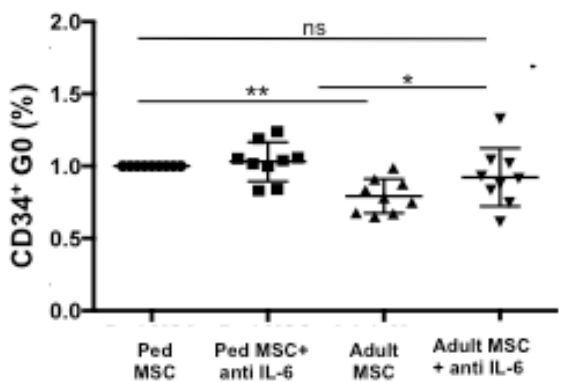

C

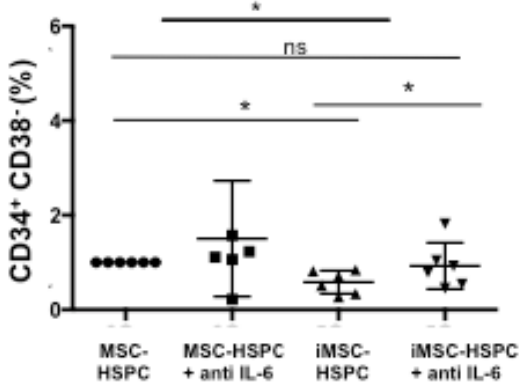

Figure 6: Increased production of IL-6 by senescent and aged MSC impairs HSPC quiescence. A. CD34 ${ }^{+}$proliferation, $\mathbf{B}$. percentage of $\mathrm{CD}_{3} 4^{+}$in generation 0 , and $\mathbf{C}$. frequency of $\mathrm{CD} 34^{+} \mathrm{CD} 38^{-}$subpopulations were determined after 4 days in co-cultures with irradiated ( $5 \mathrm{~Gy}$ ) and non-irradiated MSC in the presence or absence of an IL-6 neutralizing antibody (20ng/ml). Similar experiments were performed comparing the effect of IL-6 neutralization in adult and pediatric MSC-HSPC co-cultures D.-F. Results were normalized to 0 Gy control in A. -C. and Ped MSC condition in D.-F.. Mean value \pm SD of 10 independent experiments are reported where (*) indicates $p$ $\leq 0.05 ;(* *)$ indicates $p \leq 0.01 ;(\mathrm{ns})$ indicates $p>0.05$. 
Table 1: Demographics and clinical features of the adult and pediatric MSC donors

\begin{tabular}{|c|c|c|}
\hline & \begin{tabular}{|l} 
Adult donors \\
$(n=19)$
\end{tabular} & $\begin{array}{l}\text { Pediatric Donors } \\
(n=17)\end{array}$ \\
\hline \multicolumn{3}{|l|}{ Demographics } \\
\hline Sex ( $\%$ female/ male $)$ & $36 / 64$ & $53 / 47$ \\
\hline Age (mean \pm SD years) & $59.4 \pm 12.9$ & $13.9 \pm 2.8$ \\
\hline \multicolumn{3}{|l|}{ Surgical Procedure (\%) } \\
\hline Cardiovascular & 100 & - \\
\hline Orthopaedic & - & 100 \\
\hline \multicolumn{3}{|c|}{ Clinical Characteristics (\%) } \\
\hline Tobacco Use & 40 & - \\
\hline Diabetes & 42 & - \\
\hline \multicolumn{3}{|l|}{ Medications (\%) } \\
\hline Statins & 64 & - \\
\hline Aspirin & 72 & - \\
\hline Metformin & 36 & - \\
\hline
\end{tabular}

[39]. Furthermore, multiple myeloma -MSC derived from deceased patients displayed features of cellular senescence such as SABG activity and increased their hematopoietic support capacity, suggesting that besides their role in bone marrow aging, senescent and dysfunctional MSC can also influence cancerous tissue microenvironments [40].

Overall, our results suggest that the decrease in HSPC quiescence and $\mathrm{CD} 34^{+} \mathrm{CD} 38^{-}$subpopulations is linked to the increased in senescence-associated IL-6 production by senescent/adult MSC. A neutralizing antibody against IL-6 rescues HSPC quiescence and restores the frequency of the $\mathrm{CD} 34^{+} \mathrm{CD} 38^{-}$subpopulation to levels similar to those in control/pediatric co-cultures. Thus, IL-6, a potent hematopoietin that promotes myeloid differentiation and suppresses lymphoid differentiation, could induce HSPC differentiation at the expense of self-renewal and quiescence. This could contribute to the defective maintenance of primitive stem cells in the context of age-associated pathologies and the increased proliferation of cancer cells potentially using this defective microenvironment as a source of growth signals.

Overall the findings from this study demonstrate that age related changes in MSC directly affect critical properties of HSPC. Both senescent MSC and adult MSC produce elevated levels of IL-6 which decreases HSPC quiescence and reduce $\mathrm{CD} 34^{+} \mathrm{CD} 38^{-}$subpopulations. Antagonization of IL-6 'rejuvenates' adult and senescent MSC, making them functionally similar to pediatric MSC.

\section{MATERIALS AND METHODS}

\section{Patients}

This study was approved by the McGill UniversityEthics Board. Written consent was obtained from all participants. Adipose tissue samples were obtained from
19 adult donors undergoing cardiovascular surgery and 17 pediatric donors undergoing orthopedic surgery. Table 1 summarizes the demographic information of all study participants.

\section{Isolation and characterization of MSC}

MSC were isolated from adipose tissue as previously described [41] with minor modifications. Briefly following enzymatic digestion, cells were plated in $75 \mathrm{~cm}^{2}$ flasks ( $1 \mathrm{~g}$ tissue $/ 75 \mathrm{~cm}^{2}$ flask) and cultured at $37^{\circ} \mathrm{C}$ in a $5 \% \mathrm{CO}_{2}$ incubator. Adherent cells were maintained in low glucose DMEM (Wisent Inc, cat 319-010-CL) supplemented with 10\% MSC Qualified FBS (Gibco, ref $12662-029)$ and 1\% Pen Strep (Gibco, ref 15140-122) until they reached $80 \%$ confluence, at which point they were trypsinized (Corning Cellgro, ref 25-053-CI) and reseeded at a density of 5000 cells $/ \mathrm{cm}^{2}$. Isolated MSC were characterized fulfilling the minimal criteria proposed by the International Society for Cellular Therapy (ISCT) [12]. Cell surface markers were evaluated by FACS at the end of passage 2 with fluorescence conjugated anti-human antibodies all from BD biosciences: CD73-PE, APCCD34, FITC-CD90, CD105-PECYP CY5.5, CD19-APC, HLADR-APC, CD14-PERP CY5,5, and CD45-FITC. At passage 3, MSC tri-lineage differentiation was tested using the adipogenesis/ chondrogenesis differentiation kit (StemPro Cat \# A1007101), and osteogenesis differentiation kit (StemPro Cat \# A1007201) according to the manufacturers instructions. After 3 weeks, cells were fixed with $3.7 \%$ paraformaldehyde and osteoblasts were stained with Alizarin Red S (Alizarin Red S, Certified, Electron Microscopy Sciences, Product \#10360(EM)), chondrocytes with Safranin-O, (Safranin O, Certified, Electron Microscopy Sciences, Product \# 20800 (EM)) and adipocytes with Oil Red (Oil Red O, Electron Microscopy Sciences, Product \# 19056 (EM)). 


\section{CD34-based HSPC isolation}

HSPC were isolated from non-mobilized peripheral blood by magnetic cell sorting (auto-MACs) using the CD34 Microbead Kit (Miltenyi Biotec \# 130-046-702) as previously described $[42,43]$.

\section{MSC-HSPC co-cultures}

At passage 3 MSC were trypsinized and seeded in a 96 well plate $\left(9000 \mathrm{cells} / \mathrm{cm}^{2}\right)$. Twenty-four hours later the medium was changed to a serum free medium (Stem Span H3000, Stem Cell Technologies, Vancouver, BC). After 2 days of culture in serum free medium, freshly isolated CD34 cells were stained with carboxyfluorescein succimmidyl ester (CFSE) as previously described [42, 43], suspended in Stem Span H3000 (Stem Cell Technologies, Vancouver, BC), added to the allogeneic MSC cultures in a 1:1 ratio and co-cultured for 4 days.

\section{IL-6 neutralization}

A neutralizing antibody against IL-6 (RD Systems cat \# MAB206) or IgG isotype (control) was added to cocultures at day 0 and day 2 at a concentration of $20 \mathrm{ng} / \mathrm{ml}$.

\section{Flow cytometry analysis}

At day 4 of co-culture, HSPC and MSC were trypsinized and stained with CD34-APC, CD38-PE, and 7AAD. Flow cytometry (BD LSR Fortessa) data was analyzed with the FlowJo analysis software (Tree Star, USA). The fold-expansion of the overall HSPC culture (expansion index) was calculated with FlowJo. MSC size was expressed as the median signal intensity of FSC-A readings.

\section{MSC irradiation}

MSC were treated with 5 Gray (Gy) or otherwise indicated doses of gamma irradiation using GammaCell 22 and placed in a 6 well plate $\left(5000\right.$ cells $\left./ \mathrm{cm}^{2}\right)$ in complete DMEM. Four days post irradiation the cells were cocultured with HSPC (see above).

\section{Immunofluorescence}

Cells were seeded on glass slides $\left(0.70 \mathrm{~cm}^{2}\right.$ or $1.70 \mathrm{~cm}^{2}$ ) and fixed at indicated times in $10 \%$ fixation last 10 minutes followed by permeabilization last 30 minutes in $0.5 \%$ Triton X-100 (Sigma cat\#93443) in phosphatebuffered saline (PBS). Subsequently, cells were incubated in blocking solution (1\% BSA, IgG free, protease free,
4\% Normal Donkey Serum (Jackson ImmunoReaseach cat\#001-000-162, Sigma cat\#D966) for $60 \mathrm{~min}$ prior to incubation with primary antibodies over night at $4{ }^{\circ} \mathrm{C}$ (53BP1: Novus biologicals cat\#NB 100-304, PML: Santa Cruz cat\#sc-9862, $\gamma$ H2AX: Upstate cat\#05636). Cells were then washed with PBS and incubated with indicated secondary antibodies for 60 minutes (Life technologies Alexa Fluor anti-goat $/$ mouse/rabbit $488 \mathrm{~nm} / 594 \mathrm{~nm}$ ). The slides were finally washed, counterstained with Hoechst and mounted with Vectashield (Vector Laboratories cat\#H-1000). Images were acquired using a Axioobserver Zi Zeiss Scanning Microscope and processed/analyzed using Photoshop CS5 and the Axiovision software (Assay builder).

\section{Senescence-associated beta-galactosidase (SABG) staining}

Non-irradiated (control) and 5Gy irradiated MSC were counted and plated $24 \mathrm{~h}$ post irradiation. SABG activity was assessed 8 days post-irradiation using previously described protocols [19].

\section{ELISA}

MSC were plated in 96 well plates $(2500$ cells/ well) and supernatants were collected at day 2-4 post seeding in serum free medium (Stem Span H3000, Stem Cell Technologies, Vancouver, BC) and stored at -80C. Quantification of IL-6 and IL-8 was done with ELISA (Human IL-6 kit (BD OptEIA cat\#555220) and Human IL-8 kit (BD OptEIA cat\#555244) according to the manufacturers protocol. ELISAs were performed in triplicates and results were normalized to cell number (DRAQ5 intensity).

\section{DNA staining (LICOR)}

Cells were seeded in 96-well plates (Clear black, Greiner Bio one 655090) at 1000 cells per well and cultured/treated as indicated. At selected experimental points, plates/cells were washed, fixed, and incubated with a DRAQ5 solution (5mM Cell Signaling \#4084, diluted 1:10000). Following the final wash the plates were scanned using a LI-COR Odyssey system. The data from digitized signal intensity was processed using the Trim Mean option in the Image Studio 3 software.

\section{Statistical analyses}

Summary data is presented as mean \pm standard deviation. Wilcoxon matched-pairs signed rank test was used to assess differences in the MSC irradiation experiments, whereas Mann-Whitney test was used for 
the comparisons between the adult and pediatric MSC. All analyses were performed using the GraphPad Prism software (Graph-Pad, San Diego, USA). All hypotheses tests were 2 -sided and significance was set at the 0.05 level.

\section{ACKNOWLEDGMENTS}

This work was supported by grants from the Canadian Institutes of Health Research (CIHR grant MOP125857 to IC; CIHR grant MOP114962 to FR), Fonds de Recherche Québec Santé (FRSQ grant 29274 to IC; FRSQ grant 22624 to FR), Institut du cancer de Montréal (FR), Research Institute McGill University (IC), Institut du cancer de Montréal Canderel student fellowship (SN).

\section{CONFLICTS OF INTEREST}

There is no conflict of interest.

\section{REFERENCES}

1. Mercier FE, Ragu C and Scadden DT. The bone marrow at the crossroads of blood and immunity. Nat Rev Immunol. $2011 ; 12: 49-60$

2. Mendelson A and Frenette PS. Hematopoietic stem cell niche maintenance during homeostasis and regeneration. Nat Med. 2014; 20:833-846.

3. Anthony BA and Link DC. Regulation of hematopoietic stem cells by bone marrow stromal cells. Trends Immunol. 2014; 35:32-37.

4. Bartling B, Koch A, Simm A, Scheubel R, Silber RE and Santos AN. Insulin-like growth factor binding proteins-2 and -4 enhance the migration of human CD34-/CD133+ hematopoietic stem and progenitor cells. Int J Mol Med. 2010; 25:89-96.

5. Larochelle A, Krouse A, Metzger M, Orlic D, Donahue RE, Fricker S, Bridger G, Dunbar CE and Hematti P. AMD3100 mobilizes hematopoietic stem cells with longterm repopulating capacity in nonhuman primates. Blood. 2006; 107:3772-3778.

6. Baker DJ, Wijshake T, Tchkonia T, LeBrasseur NK, Childs BG, van de Sluis B, Kirkland JL and van Deursen JM. Clearance of p16Ink4a-positive senescent cells delays ageing-associated disorders. Nature. 2011; 479:232-236.

7. Fei C, Zhao Y, Guo J, Gu S, Li X and Chang C. Senescence of bone marrow mesenchymal stromal cells is accompanied by activation of $\mathrm{p} 53 / \mathrm{p} 21$ pathway in myelodysplastic syndromes. Eur J Haematol. 2014; 93:476-486.

8. Chang J, Wang Y, Shao L, Laberge RM, Demaria M, Campisi J, Janakiraman K, Sharpless NE, Ding S, Feng W, Luo Y, Wang X, Aykin-Burns N, Krager K, Ponnappan $\mathrm{U}$, Hauer-Jensen M, et al. Clearance of senescent cells by
ABT263 rejuvenates aged hematopoietic stem cells in mice. Nat Med. 2016; 22:78-83.

9. van Deursen JM. The role of senescent cells in ageing. Nature. 2014; 509:439-446.

10. Coppe JP, Patil CK, Rodier F, Sun Y, Munoz DP, Goldstein J, Nelson PS, Desprez PY and Campisi J. Senescenceassociated secretory phenotypes reveal cell-nonautonomous functions of oncogenic RAS and the p53 tumor suppressor. PLoS Biol. 2008; 6:2853-2868.

11. Rodier F and Campisi J. Four faces of cellular senescence. J Cell Biol. 2011; 192:547-556.

12. Dominici M, Le Blanc K, Mueller I, Slaper-Cortenbach I, Marini F, Krause D, Deans R, Keating A, Prockop D and Horwitz E. Minimal criteria for defining multipotent mesenchymal stromal cells. The International Society for Cellular Therapy position statement. Cytotherapy. 2006; $8: 315-317$

13. Rodier F, Coppe JP, Patil CK, Hoeijmakers WA, Munoz DP, Raza SR, Freund A, Campeau E, Davalos AR and Campisi J. Persistent DNA damage signalling triggers senescence-associated inflammatory cytokine secretion. Nat Cell Biol. 2009; 11:973-979.

14. Rodier F, Munoz DP, Teachenor R, Chu V, Le O, Bhaumik D, Coppe JP, Campeau E, Beausejour CM, Kim SH, Davalos AR and Campisi J. DNA-SCARS: distinct nuclear structures that sustain damage-induced senescence growth arrest and inflammatory cytokine secretion. J Cell Sci. 2011; 124:68-81.

15. d'Adda di Fagagna F, Reaper PM, Clay-Farrace L, Fiegler H, Carr P, Von Zglinicki T, Saretzki G, Carter NP and Jackson SP. A DNA damage checkpoint response in telomere-initiated senescence. Nature. 2003; 426:194-198.

16. Ward IM, Minn K, Jorda KG and Chen J. Accumulation of checkpoint protein 53BP1 at DNA breaks involves its binding to phosphorylated histone H2AX. J Biol Chem. 2003; 278:19579-19582.

17. Pearson M, Carbone R, Sebastiani C, Cioce M, Fagioli M, Saito S, Higashimoto Y, Appella E, Minucci S, Pandolfi PP and Pelicci PG. PML regulates p53 acetylation and premature senescence induced by oncogenic Ras. Nature. 2000; 406:207-210.

18. Malaquin N, Carrier-Leclerc A, Dessureault M and Rodier F. DDR-mediated crosstalk between DNA-damaged cells and their microenvironment. Front Genet. 2015; 6:94.

19. Stenderup K, Justesen J, Clausen C and Kassem M. Aging is associated with decreased maximal life span and accelerated senescence of bone marrow stromal cells. Bone. 2003; 33:919-926.

20. Choudhery MS, Badowski M, Muise A, Pierce J and Harris DT. Donor age negatively impacts adipose tissue-derived mesenchymal stem cell expansion and differentiation. J Transl Med. 2014; 12:8.

21. Pirih FQ, Michalski MN, Cho SW, Koh AJ, Berry JE, Ghaname E, Kamarajan P, Bonnelye E, Ross CW, 
Kapila YL, Jurdic P and McCauley LK. Parathyroid hormone mediates hematopoietic cell expansion through interleukin-6. PLoS One. 2010; 5:e13657.

22. Kuranda K, Vargaftig J, de la Rochere P, Dosquet C, Charron D, Bardin F, Tonnelle C, Bonnet D and Goodhardt M. Age-related changes in human hematopoietic stem/ progenitor cells. Aging Cell. 2011; 10:542-546.

23. Song Z, Zhang J, Ju Z and Rudolph KL. Telomere dysfunctional environment induces loss of quiescence and inherent impairments of hematopoietic stem cell function. Aging Cell. 2012; 11:449-455.

24. Siegel G, Kluba T, Hermanutz-Klein U, Bieback K, Northoff $\mathrm{H}$ and Schafer R. Phenotype, donor age and gender affect function of human bone marrow-derived mesenchymal stromal cells. BMC Med. 2013; 11:146.

25. Kizilay Mancini O, Shum-Tim D, Stochaj U, Correa JA and Colmegna I. Age, atherosclerosis and type 2 diabetes reduce human mesenchymal stromal cell-mediated T-cell suppression. Stem Cell Res Ther. 2015; 6:140.

26. Cmielova J, Havelek R, Soukup T, Jiroutova A, Visek B, Suchanek J, Vavrova J, Mokry J, Muthna D, Bruckova L, Filip S, English D and Rezacova M. Gamma radiation induces senescence in human adult mesenchymal stem cells from bone marrow and periodontal ligaments. Int J Radiat Biol. 2012; 88:393-404.

27. Sepulveda JC, Tome M, Fernandez ME, Delgado M, Campisi J, Bernad A and Gonzalez MA. Cell senescence abrogates the therapeutic potential of human mesenchymal stem cells in the lethal endotoxemia model. Stem Cells. 2014; 32:1865-1877.

28. Yoon DS, Kim YH, Lee S, Lee KM, Park KH, Jang Y and Lee JW. Interleukin-6 induces the lineage commitment of bone marrow-derived mesenchymal multipotent cells through down-regulation of Sox 2 by osteogenic transcription factors. FASEB J. 2014; 28:3273-3286.

29. Walter D, Lier A, Geiselhart A, Thalheimer FB, Huntscha S, Sobotta MC, Moehrle B, Brocks D, Bayindir I, Kaschutnig P, Muedder K, Klein C, Jauch A, Schroeder T, Geiger H, Dick TP, et al. Exit from dormancy provokes DNA-damage-induced attrition in haematopoietic stem cells. Nature. 2015; 520:549-552.

30. Ju Z, Jiang H, Jaworski M, Rathinam C, Gompf A, Klein C, Trumpp A and Rudolph KL. Telomere dysfunction induces environmental alterations limiting hematopoietic stem cell function and engraftment. Nat Med. 2007; 13:742-747.

31. Xiao M and Dooley DC. Cellular and molecular aspects of human CD34+ CD38- precursors: analysis of a primitive hematopoietic population. Leuk Lymphoma. 2000; 38:489497.

32. Adams PD, Jasper H and Rudolph KL. Aging-Induced Stem Cell Mutations as Drivers for Disease and Cancer. Cell Stem Cell. 2015; 16:601-612.

33. $\mathrm{Wu} \mathrm{W}$, Niklason $\mathrm{L}$ and Steinbacher DM. The effect of age on human adipose-derived stem cells. Plast Reconstr Surg.
2013; 131:27-37.

34. Tchkonia T, Zhu Y, van Deursen J, Campisi J and Kirkland JL. Cellular senescence and the senescent secretory phenotype: therapeutic opportunities. J Clin Invest. 2013; 123:966-972.

35. Demaria M, Ohtani N, Youssef SA, Rodier F, Toussaint W, Mitchell JR, Laberge RM, Vijg J, Van Steeg H, Dolle ME, Hoeijmakers JH, de Bruin A, Hara E and Campisi J. An essential role for senescent cells in optimal wound healing through secretion of PDGF-AA. Dev Cell. 2014; 31:722733.

36. Laberge RM, Sun Y, Orjalo AV, Patil CK, Freund A, Zhou L, Curran SC, Davalos AR, Wilson-Edell KA, Liu S, Limbad C, Demaria M, Li P, Hubbard GB, Ikeno Y, Javors $\mathrm{M}$, et al. MTOR regulates the pro-tumorigenic senescence-associated secretory phenotype by promoting IL1A translation. Nat Cell Biol. 2015; 17:1049-1061.

37. Cheng $S$ and Rodier F. Manipulating senescence in health and disease: emerging tools. Cell Cycle. 2015; 14:16131614.

38. Walenda T, Bork S, Horn P, Wein F, Saffrich R, Diehlmann A, Eckstein V, Ho AD and Wagner W. Co-culture with mesenchymal stromal cells increases proliferation and maintenance of haematopoietic progenitor cells. J Cell Mol Med. 2010; 14:337-350.

39. Chakkalakal JV, Jones KM, Basson MA and Brack AS. The aged niche disrupts muscle stem cell quiescence. Nature. 2012; 490:355-360.

40. Andre T, Meuleman N, Stamatopoulos B, De Bruyn C, Pieters K, Bron D and Lagneaux L. Evidences of early senescence in multiple myeloma bone marrow mesenchymal stromal cells. PLoS One. 2013; 8:e59756.

41. Bernacki SH, Wall ME and Loboa EG. Isolation of human mesenchymal stem cells from bone and adipose tissue. Methods Cell Biol. 2008; 86:257-278.

42. Colmegna I, Pryshchep S, Oishi H, Goronzy JJ and Weyand CM. Dampened ERK signaling in hematopoietic progenitor cells in rheumatoid arthritis. Clin Immunol. 2012; 143:7382.

43. Colmegna I, Diaz-Borjon A, Fujii H, Schaefer L, Goronzy JJ and Weyand CM. Defective proliferative capacity and accelerated telomeric loss of hematopoietic progenitor cells in rheumatoid arthritis. Arthritis Rheum. 2008; 58:9901000 . 